\title{
In Situ Liquid Cell Electron Microscopy: An Evolving Tool for Biomedical and Life Science Applications
}

\author{
Madeline J. Dukes ${ }^{1}$, A. Cameron Varano ${ }^{2,3}$ and Deborah F. Kelly ${ }^{2}$ \\ 1. Application Science, Protochips, Inc., Morrisville, NC 27560, USA \\ 2. Virginia Tech Carilion Research Institute, Virginia Tech, Roanoke, VA 24016, USA. \\ 3. Translational Biology, Medicine, and Health Graduate Program, Virginia Tech, Blacksburg, VA \\ 24061, USA.
}

During the last decade, Liquid Cell-Transmission Electron Microscopy (LC-TEM) has proved to be an increasingly valuable tool in the microscopist's repertoire. LC-TEM techniques involve sandwiching a thin layer of liquid between two ultrathin electron transparent membranes, typically either silicon nitride or graphene. These membranes are contained within specially designed sample holders that hermetically seal the contained liquid from the vacuum of the TEM column. Thus, researchers have been able to observe dynamic processes and behaviors at nanoscale resolutions in their native liquid environment and take advantage of the powerful analytical tools available to the electron microscope [1]. Commercially available LC-TEM holders have an increasingly sophisticated suite of options available, including the capability to heat and maintain a given temperature or deliver electrical stimuli directly to the sample. During real-time imaging, processes such as particle growth, interactions, and fine movements can be observed at the nanoscale. Already widely embraced by the materials fields to observe nanomaterial properties and electrochemical mechanisms, LC-TEM also represents a growing opportunity to transform our understanding of many biological events.

Using the Protochips Poseidon Select in situ liquid TEM holder, we have recently observed mobile processes of biological specimens in a native liquid environment that mimics the human body. Spatial displacement of the sample within the liquid holder due to diffusion can limit the interpretation of fine molecular movements. To alleviate this issue without arresting the intrinsic mobility of the specimen of interest, we employed two strategies. First, we utilized microwells etched onto the surface of the silicon nitride membrane to limit the long-range diffusion of the sample within the viewing area. Secondly, we employed affinity chemistry techniques to tether samples to the surface of the membrane [2]. Using this combinatorial approach, we were able to observe real-time dynamic movements in enzymatically active double layer rotavirus particles in a native liquid environment (Figure 1). We also employed similar strategies to image the real-time interactions between therapeutic nanoparticles and eukaryotic cells as shown in Figure 2 [3]. Our results demonstrate that nanoparticle movement across cellular membranes can be observed in situ, enabling researchers to gain a clearer understanding of a nanomaterial interactions within a physiological, liquid environment.

Drug delivery and clinical therapy development require robust methods to view dynamic systems in realtime. LC-TEM provides a critical opportunity to visualize these dynamic processes. Overall, LC-TEM, has the potential to transform the way we view nanoscale processes by allowing us to realistically observe the nanoworld around us.

\section{References:}

[1] Demmert et al., in (2016) 'Visualizing Macromolecules in Liquid at the Nanoscale', in Ross, F.M. (ed.) Liquid Cell Electron Microscopy. Cambridge: Cambridge University Press, pp. 334-355.

[2] Varano et al., Chem. Commun., 2015, 51, 16176-16179.

[3] Pohlmann et al., Nano Letters, 15.4 (2015): 2329-2335. 


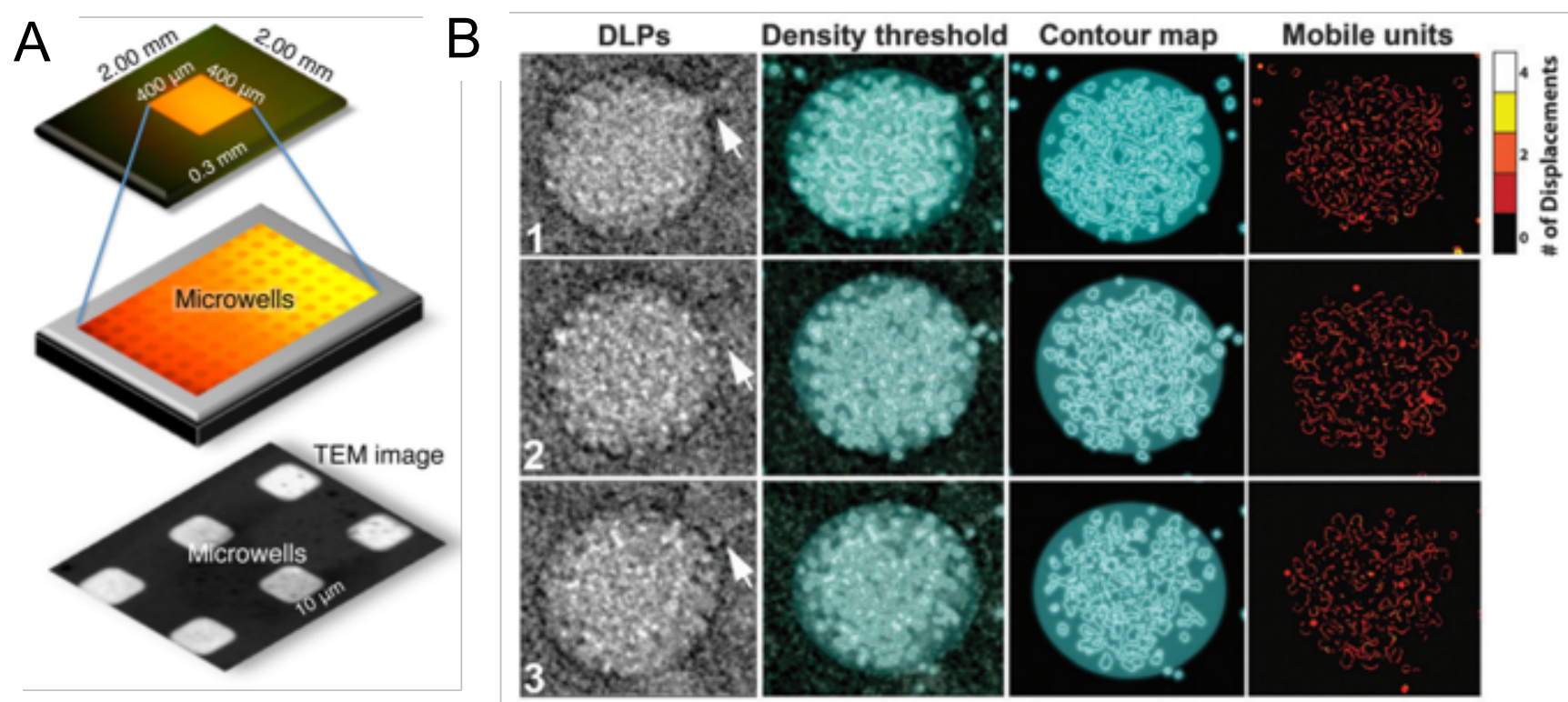

Figure 1. LC-TEM quantitative analysis of mobile double-layered rotavirus particles (DLPs). (A) Schematic of microwells etched onto the surface of the silicon nitride membrane. Rotavirus DLPs are tethered within individual microwells to prevent long range diffusion in the liquid cell. (B) Contour mapping of individual transcriptionally-active DLPs during real-time image acquisition. Images adapted from references [1] and [2].
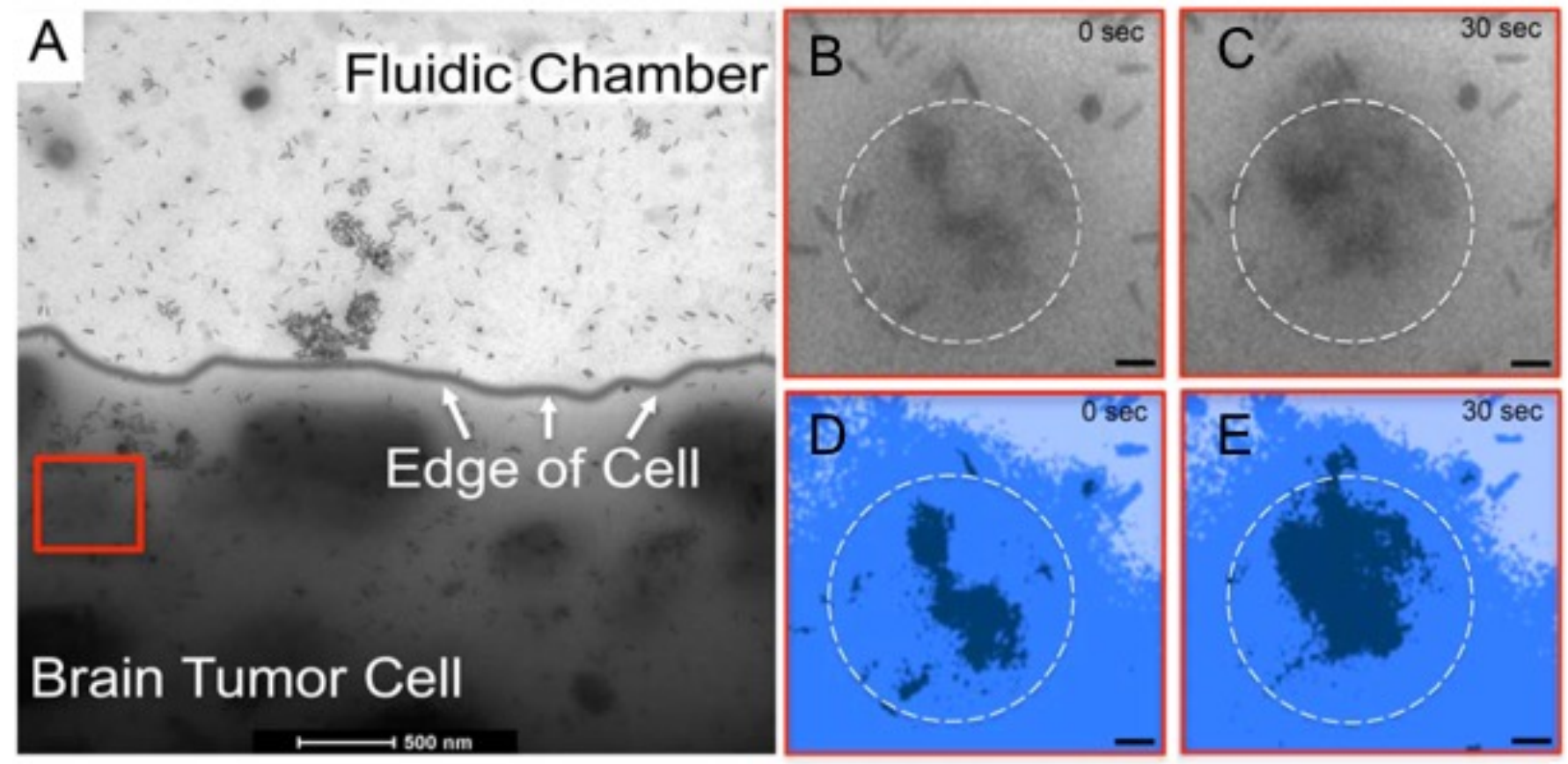

Figure 2. Internalization of gold nanorods after a 60-minute incubation with brain tumor cells. (A) Edge of the cell in the fluidic chamber. (B) The region highlighted by the red box in A at time zero and (C) thirty seconds. (D) The same regions presented as a contour maps for time zero and (E) thirty seconds shows the accumulation of gold nanorods over time. Image adapted from reference [3]. 